PROCEEDINGS OF THE

AMERICAN MATHEMATICAL SOCIETY

Volume 137, Number 9, September 2009, Pages 2849-2856

S 0002-9939(09)09835-9

Article electronically published on March 4, 2009

\title{
FROM A RAMANUJAN-SELBERG CONTINUED FRACTION TO A JACOBIAN IDENTITY
}

\author{
HEI-CHI CHAN
}

(Communicated by Ken Ono)

\begin{abstract}
Jacobi proved an elegant identity involving eight-fold infinite products. In this paper, we give a new proof of this identity. A key ingredient of our proof is an identity satisfied by a Ramanujan-Selberg continued fraction.
\end{abstract}

\section{INTRODUCTION}

For $|q|<1$, we define

$$
T(q):=\frac{1}{1}+\frac{q}{1}+\frac{q+q^{2}}{1}+\frac{q^{3}}{1}+\frac{q^{2}+q^{4}}{1}+\cdots
$$

Independently, Ramanujan and Selberg studied this interesting continued fraction. Ramanujan asserted in his notebook [25, p. 290] that

$$
T(q)=\frac{\left(-q^{2} ; q^{2}\right)_{\infty}}{\left(-q ; q^{2}\right)_{\infty}}
$$

Here and in the rest of the paper we follow the customary $q$-product notation: we set (for $|q|<1$ )

$$
(c ; q)_{\infty}:=\prod_{k=0}^{\infty}\left(1-c q^{k}\right) .
$$

It was A. Selberg, unaware of Ramanujan's assertion at the time of writing, who gave the first proof of (1.1) in print [26]. Other proofs were discovered by Ramanathan [24], Andrews, Berndt, Jacobsen and Lamphere [4], and, Zhang [30]. Zhang, building upon his earlier results in 31, 32, gave explicit evaluations of $T(q)$ in terms of class invariants and singular moduli in 33 . See also the paper by Baruah and Saikia [7] for a penetrating study of $T(q)$ (and related continued fractions).

It may not be obvious how $T(q)$ is related to the following amazing identity discovered by Jacobi:

Theorem 1.1 (Jacobi). For $|q|<1$,

$$
\left(q ; q^{2}\right)_{\infty}^{8}+16 q\left(-q^{2} ; q^{2}\right)_{\infty}^{8}=\left(-q ; q^{2}\right)_{\infty}^{8}
$$

Received by the editors October 9, 2008, and, in revised form, November 24, 2008.

2000 Mathematics Subject Classification. Primary 05A15, 05A30, 05A40.

Key words and phrases. Ramanujan-Selberg continued fraction, Jacobian identity.

(C)2009 American Mathematical Society 
or, in standard notation,

$$
\prod_{n=1}^{\infty}\left(1-q^{2 n-1}\right)^{8}+16 q \prod_{n=1}^{\infty}\left(1+q^{2 n}\right)^{8}=\prod_{n=1}^{\infty}\left(1+q^{2 n-1}\right)^{8} .
$$

Whittaker and Watson note that [29, p. 470] Jacobi was deeply impressed by (1.2). For proofs of (1.2), see the wonderful books by Whittaker and Watson 29] and by the Borweins 11; see also an elegant paper by Ewell 19.

In this paper, we will give a new proof of (1.2) that is based on the following identity of $T(q)$ :

Theorem 1.2. Let $x(q)=1 /\left(2 T^{2}(q)\right)$. Then

$$
x(q)-\frac{q^{1 / 2}}{x(q)}=\frac{\left(q^{1 / 2} ; q^{1 / 2}\right)_{\infty}^{4}}{2\left(q^{2} ; q^{2}\right)_{\infty}^{4}} .
$$

In Section 2, we will prove Theorem 1.2, In Section 3, we will prove the Jacobian Identity (1.2). Section 4 is our concluding remarks.

Before we turn to Section 2, we apply Theorem 1.2 to evaluate $T(q)$. In terms of the eta function $\eta(z)=e^{2 \pi i z / 24} \prod_{n=1}\left(1-e^{2 \pi i n z}\right)$, we can write (1.3) as (with $\left.q=e^{2 \pi i z}\right)$

$$
x(q)-\frac{q^{1 / 2}}{x(q)}=\frac{1}{2} q^{1 / 4}\left(\frac{\eta(z / 2)}{\eta(2 z)}\right)^{4} .
$$

With the well-known identity, $\eta(-1 / z)=\sqrt{z / i} \eta(z)$, we have, at $z=i$ (i.e., $q=$ $\left.e^{-2 \pi}\right), \eta(i / 2) / \eta(2 i)=\sqrt{2}$. This, with (1.4), implies

$$
T\left(e^{-2 \pi}\right)=\sqrt{\frac{e^{\pi / 2}}{2(\sqrt{2}+1)}} .
$$

For a comprehensive theory of the evaluations of $T(q)$, consult the wonderful papers by Zhang [33] and by Baruah and Saikia [7.

\section{Proof of Theorem 1.2}

Our proof is motivated by the recent works [15, 16]. First, we observe that

$$
T^{2}(q)=\frac{1}{2}\left(\frac{\sum q^{j^{2}+j}}{\sum q^{j^{2}}}\right)
$$

where in both sums $j$ runs from $-\infty$ to $\infty$. To see this, we note that

$$
T^{2}(q)=\frac{1}{2}\left(\frac{2\left(q^{2} ; q^{2}\right)_{\infty}\left(-q^{2} ; q^{2}\right)_{\infty}^{2}}{\left(q^{2} ; q^{2}\right)_{\infty}\left(-q ; q^{2}\right)_{\infty}^{2}}\right)=\frac{1}{2}\left(\frac{\sum q^{j^{2}+j}}{\sum q^{j^{2}}}\right) .
$$

The first equation is due to (1.1). The second is from Jacobi's Triple Product Identity (see, e.g., 2, 3, 6, 9])

$$
\sum_{n=-\infty}^{\infty}(-z)^{n} Q^{n^{2}}=\prod_{j=1}^{\infty}\left(1-Q^{2 j}\right)\left(1-z Q^{2 j-1}\right)\left(1-z^{-1} Q^{2 j-1}\right) .
$$

This proves (2.1). 
For later convenience, we define, for $a=0$ and 1 ,

$$
G(a):=\sum_{j=-\infty}^{\infty} q^{j^{2}+a j}
$$

and

$$
J(z):=\prod_{n=1}^{\infty}\left(1-q^{n / 2}\right)\left(1+z q^{(2 n-1) / 4}\right)\left(1+z^{-1} q^{(2 n-1) / 4}\right) .
$$

Note that the dependence of $G$ on $q$ is suppressed in this notation. Also, we can write $x(q)=1 /\left(2 T^{2}(q)\right)$ (as defined in Theorem 1.2) as

$$
x(q)=\frac{G(0)}{G(1)} .
$$

With the above notation understood, we prove the following lemma, which expresses $G$ in terms of $J( \pm 1)$ :

\section{Lemma 2.1.}

$$
\begin{aligned}
G(0) & =\frac{1}{2}(J(1)+J(-1)), \\
q^{1 / 4} G(1) & =\frac{1}{2}(J(1)-J(-1)) .
\end{aligned}
$$

Proof. We start with defining, for $a=0$ and 1,

$$
K_{n}(a):=\sum_{j=-\infty}^{\infty} q^{j^{2}+a j}\left[\begin{array}{c}
2 n \\
n+2 j
\end{array}\right] .
$$

Here, the $q$-binomial coefficients (or the Gaussian polynomials) are defined as

$$
\left[\begin{array}{c}
A \\
B
\end{array}\right]=\left\{\begin{array}{cl}
0 & \text { if } B<0 \text { or } B>A \text { or } A<0 \\
\frac{\left(1-q^{A}\right)\left(1-q^{A-1}\right) \cdots\left(1-q^{A-B+1}\right)}{\left(1-q^{B}\right)\left(1-q^{B-1}\right) \cdots(1-q)} & \text { otherwise. }
\end{array}\right.
$$

See, e.g., $[2$, 6]. We will need the following property of the $q$-binomial numbers (see, e.g., [6]): for fixed $m_{1}$ and $m_{2}$, with $R>S$ positive,

$$
\lim _{N \rightarrow \infty}\left[\begin{array}{c}
R N+m_{1} \\
S N+m_{2}
\end{array}\right]=\frac{1}{(q ; q)_{\infty}} .
$$

The key idea of our proof is to express (the limit of) $K_{n}(a)$ in two different ways.

First, we note that, with $K_{\infty}(a):=\lim _{n \rightarrow \infty} K_{n}(a)$, we have, by (2.8) and (2.9),

$$
K_{\infty}(a)=\frac{G(a)}{(q ; q)_{\infty}} .
$$

Next, we write $K_{\infty}(a)$ in a different way. To proceed, we observe that

$$
\begin{aligned}
K_{n}(a) & =\sum_{\sigma \equiv 0(\bmod 2)} q^{\sigma^{2} / 4+(a / 2) \sigma}\left[\begin{array}{c}
2 n \\
n+\sigma
\end{array}\right] \\
& =\frac{1}{2} \sum_{k=0}^{1}\left(\sum_{\sigma=-\infty}^{\infty} q^{\sigma^{2} / 4+(a / 2) \sigma}\left[\begin{array}{c}
2 n \\
n+\sigma
\end{array}\right](-1)^{k \sigma}\right) \\
& :=\frac{1}{2} \sum_{k=0}^{1} C_{n}\left((-1)^{k}, a\right) .
\end{aligned}
$$


Note that the second line is due to the fact that

$$
\frac{1}{2} \sum_{j=0}^{1}(-1)^{j \sigma}= \begin{cases}1 & \text { if } \sigma \equiv 0(\bmod 2), \\ 0 & \text { otherwise. }\end{cases}
$$

Let $C_{\infty}(z, a):=\lim _{n \rightarrow \infty} C_{n}(z, a)$; then (2.11) implies

$$
K_{\infty}(a)=\frac{1}{2} \sum_{k=0}^{1} C_{\infty}\left((-1)^{k}, a\right) .
$$

To finish proving the second way of expressing $K_{\infty}(a)$, we need to write $C_{\infty}(z, a)$ in terms of $J(z)$. We claim that

$$
\begin{aligned}
C_{\infty}(z, 0) & =\frac{J(z)}{(q ; q)_{\infty}} \\
C_{\infty}(z, 1) & =\frac{1}{z q^{1 / 4}}\left(\frac{J(z)}{(q ; q)_{\infty}}\right) .
\end{aligned}
$$

To prove (2.14), we observe that

$$
\begin{aligned}
C_{\infty}(z, 1) & =\frac{1}{(q ; q)_{\infty}} \sum_{\sigma=-\infty}^{\infty} z^{\sigma} q^{\sigma^{2} / 4+\sigma / 2} \\
& =\frac{1}{(q ; q)_{\infty}} \prod_{n=1}^{\infty}\left(1-q^{n / 2}\right)\left(1+z q^{(2 n+1) / 4}\right)\left(1+z^{-1} q^{(2 n-3) / 4}\right) \\
& =\frac{1}{z q^{1 / 4}}\left(\frac{J(z)}{(q ; q)_{\infty}}\right) .
\end{aligned}
$$

Note that the first line follows from the definition of $C_{n}(z, a)$ (cf. (2.11)) and the property of the $q$-binomial numbers (cf. (2.9)). The second line is due to Jacobi's Triple Product Identity (cf. (2.2) ). For the last line, we recall the definition of $J(z)$ in (2.4). The proof of (2.13) is similar and will be omitted. Note that (2.12)-(2.14) give us a second way of writing $K_{\infty}(a)$.

By putting the above together, we prove the lemma. Indeed,

$$
\begin{aligned}
\frac{G(1)}{(q ; q)_{\infty}} & =\frac{1}{2} \sum_{k=0}^{1} C_{\infty}\left((-1)^{k}, 1\right) \quad \text { by (2.10) } \text { and (2.12) } \\
& =\frac{1}{2 q^{1 / 4}(q ; q)_{\infty}}(J(1)-J(-1)) \quad \text { by (2.14) }
\end{aligned}
$$

This proves (2.7). The proof of (2.6) follows the same type of calculation and will be omitted here.

Proof of Theorem [1.2. First, we write the two identities in Lemma 2.1 as

$$
\begin{aligned}
& G(0)+q^{1 / 4} G(1)=J(1), \\
& G(0)-q^{1 / 4} G(1)=J(-1) .
\end{aligned}
$$

Next, we multiply them together to get

$$
G^{2}(0)-q^{1 / 2} G^{2}(1)=J(1) J(-1) .
$$


By dividing the last equation by $G(0) G(1)$ we obtain

$$
x(q)-\frac{q^{1 / 2}}{x(q)}=\frac{J(1) J(-1)}{G(0) G(1)}
$$

(recall that $x=G(0) / G(1)$ by (2.5)). Our last task is to simplify the right-hand side of (2.15). To this end, we observe that

$$
\begin{aligned}
\frac{J(1) J(-1)}{G(0) G(1)} & =\frac{1}{2} \prod_{n=1} \frac{\left(1-q^{n / 2}\right)^{2}\left(1+q^{(2 n-1) / 4}\right)^{2}\left(1-q^{(2 n-1) / 4}\right)^{2}}{\left(1-q^{2 n}\right)^{2}\left(1+q^{2 n}\right)^{2}\left(1+q^{2 n-1}\right)^{2}} \\
& =\frac{1}{2} \prod_{n=1} \frac{\left(1-q^{n / 2}\right)^{2}}{\left(1-q^{2 n}\right)^{2}} \cdot \frac{\left(1-q^{(2 n-1) / 2}\right)^{2}}{\left(1+q^{n}\right)^{2}} \\
& =\frac{1}{2} \prod_{n=1} \frac{\left(1-q^{n / 2}\right)^{4}}{\left(1-q^{2 n}\right)^{4}},
\end{aligned}
$$

which is the right-hand side of (1.3). Note that in the first equality we used Jacobi's Triple Identity to write $G(a)$ as infinite products. This completes our proof of Theorem 1.2,

\section{A NeW PRoOf of the JaCobian IdENTITy}

The first part of our proof mimics a proof for Ramanujan's "Most Beautiful Identity" (see [3, 9, 18, 22, 23]; see also [12] for a similar identity for Ramanujan's cubic continued fraction).

By replacing $q$ by $q^{2}$ in (1.3), we obtain

$$
x\left(q^{2}\right)-\frac{q}{x\left(q^{2}\right)}=\frac{(q ; q)_{\infty}^{4}}{2\left(q^{4} ; q^{4}\right)_{\infty}^{4}} .
$$

Replacing $q$ by $-q$ in (3.1) gives

$$
x\left(q^{2}\right)+\frac{q}{x\left(q^{2}\right)}=\frac{\left(q^{2} ; q^{2}\right)_{\infty}^{4}\left(-q ; q^{2}\right)_{\infty}^{4}}{2\left(q^{4} ; q^{4}\right)_{\infty}^{4}} .
$$

Now, square (3.2) and subtract the square of (3.1). Precisely, the left-hand side gives

$$
\left(x\left(q^{2}\right)+\frac{q}{x\left(q^{2}\right)}\right)^{2}-\left(x\left(q^{2}\right)-\frac{q}{x\left(q^{2}\right)}\right)^{2}=4 q .
$$

The other side of the same expression gives

$$
\frac{\left(q^{2} ; q^{2}\right)_{\infty}^{8}\left(-q ; q^{2}\right)_{\infty}^{8}-(q ; q)_{\infty}^{8}}{4\left(q^{4} ; q^{4}\right)_{\infty}^{8}}
$$

Equating the last two expressions gives

$$
(q ; q)_{\infty}^{8}+16 q\left(q^{4} ; q^{4}\right)_{\infty}^{8}=\left(q^{2} ; q^{2}\right)_{\infty}^{8}\left(-q ; q^{2}\right)_{\infty}^{8} .
$$

Dividing both sides of the last equation by $\left(q^{2} ; q^{2}\right)_{\infty}^{8}$ gives (1.2). 


\section{Concluding Remarks}

Two remarks are in order. First, we note that Theorem 1.2 could be compared with the following results (i.e., equations (4.1) and (4.2) below).

Let $R(q)$ denote the celebrated Rogers-Ramanujan continued fraction:

$$
R(q):=\frac{q^{1 / 5}}{1}+\frac{q}{1}+\frac{q^{2}}{1}+\frac{q^{3}}{1}+\cdots
$$

It is known that $R(q)$ satisfies the following remarkable equation:

$$
\frac{1}{R(q)}-1-R(q)=\frac{f\left(-q^{1 / 5}\right)}{q^{1 / 5} f\left(-q^{5}\right)},
$$

where $f(-q):=(q ; q)_{\infty} ;$ cf. [27, 28]. See also the following excellent introductions [2, 5, 8, 10].

Recently, the following was discovered. Denote the Ramanujan cubic continued fraction by

$$
v:=\frac{q^{1 / 3}}{1}+\frac{q+q^{2}}{1}+\frac{q^{2}+q^{4}}{1}+\cdots
$$

It can be shown that

$$
\frac{1}{x_{v}(q)}-q^{1 / 3}-2 q^{2 / 3} x_{v}(q)=\frac{\left(q^{1 / 3} ; q^{1 / 3}\right)_{\infty}\left(q^{2 / 3} ; q^{2 / 3}\right)_{\infty}}{\left(q^{3} ; q^{3}\right)_{\infty}\left(q^{6} ; q^{6}\right)_{\infty}}
$$

where $x_{v}(q)=q^{-1 / 3} v$ (cf. Theorem 2 in [12; see also its implications in [13, 14]).

Second, we remark that the strategy used in this paper can also be applied to the

Ramanujan-Göllnitz-Gordon continued fraction (see p. 229 of Ramanujan's second notebook [25]; see also [1, 20, 21]; and also the paper by Chan and Huang [17, in which the authors provided a comprehensive theory of the Ramanujan-GöllnitzGordon continued fraction) and proved certain $q$-identities. We will address these results on other occasions.

\section{ACKNOWLEDGMENTS}

The author would like to thank Professors Nayandeep Deka Baruah and Bruce Berndt for their comments and encouragement. The author would also like to thank the referee for many useful comments.

\section{REFERENCES}

[1] G. E. Andrews, On q-difference equations for certain well-poised basic hypergeometric series, Quart. J. Math. Oxford Ser. (2) 19 (1968), 433-447. MR0237831 (38:6112)

[2] G. E. Andrews, The theory of partitions, Encycl. Math. and its Appl., vol. 2, G.-C. Rota, ed., Addison-Wesley, Reading, MA, 1976 (Reissued: Cambridge University Press, Cambridge, 1998). MR0557013 (58:27738)

[3] G. E. Andrews, R. Askey and R. Roy, Special functions, Cambridge University Press, Cambridge, 1999. MR:1688958 (2000g:33001)

[4] G. E. Andrews, B. C. Berndt, L. Jacobsen and R. L. Lamphere, The continued fractions found in the unorganized portions of Ramanujan's notebooks, Mem. Amer. Math. Soc. 99 (1992), no. 477. MR1124109 (93f:11008)

[5] G. E. Andrews and B. C. Berndt, Ramanujan's lost notebook, Part I, Springer-Verlag, New York, 2005. MR2135178(2005m:11001)

[6] G. E. Andrews and K. Eriksson, Integer partitions, Cambridge University Press, Cambridge, 2004. MR2122332(2006b:11125) 
[7] N. D. Baruah and N. Saikia, Modular relations and explicit values of Ramanujan-Selberg continued fractions, Int. J. Math. Math. Sci. 2006, Art. ID 54901, 1-15. MR.2251712 (2007e:11148)

[8] B. C. Berndt, Ramanujan's notebooks, Part III, Springer-Verlag, New York, 1991. MR.1117903 (92j:01069)

[9] B. C. Berndt, Number theory in the spirit of Ramanujan, American Mathematical Society, Providence, RI, 2006. MR2246314 (2007f:11001)

[10] B. C. Berndt, H. H. Chan, S.-S. Huang, S.-Y. Kang, J. Sohn, and S. H. Son, The RogersRamanujan continued fraction, J. Comput. Appl. Math. 105 (1999), 9-24. MR1690576 (2000b:11009)

[11] J. M. Borwein and P. B. Borwein, Pi and the AGM. A study in analytic number theory and computational complexity, Wiley, New York, 1987. MR877728(89a:11134)

[12] H. C. Chan, Ramanujan's cubic continued fraction and an analog of his "most beautiful identity", to appear in International Journal of Number Theory.

[13] H. C. Chan, Ramanujan's cubic continued fraction and Ramanujan type congruences for a certain partition function, to appear in International Journal of Number Theory.

[14] H. C. Chan, Distribution of a certain partition function modulo powers of primes, submitted.

[15] H. C. Chan, A new proof for two identities involving the Ramanujan's cubic continued fraction, submitted.

[16] H. C. Chan and S. Ebbing, Fractorization theorems for the Rogers-Ramanujan continued fraction in the lost notebook, submitted.

[17] H. H. Chan and S.-S. Huang, On the Ramanujan-Göllnitz-Gordon continued fraction, Ramanujan J. 1 (1997), 75-90. MR.1607529 (99k:11015)

[18] W. Chu and L. Di Claudio, Classical Partition Identities and Basic Hypergeometric Series, Università degli Studi di Lecce, Lecce, Italy, 2004.

[19] J. A. Ewell, A note on a Jacobian identity, Proc. Amer. Math. Soc. 126 (1998), 421-423. MR1451797 (98k:33030)

[20] H. Göllnitz, Partitionen mit Differenzenbedingungen, J. Reine Angew. Math. 225 (1967), 154-190. MR0211973 (35:2848)

[21] B. Gordon, Some continued fractions of the Rogers-Ramanujan type, Duke Math. J. 32 (1965), 741-748. MR0184001 (32:1477)

[22] M. D. Hirschhorn, An identity of Ramanujan, and applications, in $q$-Series from a Contemporary Perspective, Contemporary Mathematics, vol. 254, American Mathematical Society, Providence, RI, 2000, 229-234. MR1768930 (2001f:11180)

[23] M. D. Hirschhorn, Ramanujan's “most beautiful identity”, Austral. Math. Soc. Gazette 32 (2005), 259-262. MR2176248 (2006e:11158)

[24] K. G. Ramanathan, Ramanujan's continued fraction, Indian J. Pure Appl. Math. 16 (1985), 695-724. MR801801 (87e:11015)

[25] S. Ramanujan, Notebooks (2 volumes), Tata Institute of Fundamental Research, Bombay, 1957. MR0099904 (20:6340)

[26] A. Selberg, Uber einige arithmetische Identitäten, Avh. Norske Vid.-Akad. Oslo I. Mat.Naturv. Kl. 8 (1936), 3-23.

[27] G. N. Watson, Theorems stated by Ramanujan (VII): Theorems on continued fractions, J. London Math. Soc. 4 (1929), 39-48.

[28] G. N. Watson, Theorems stated by Ramanujan (IX): Two continued fractions, J. London Math. Soc. 4 (1929), 231-237.

[29] E. T. Whittaker and G. N. Watson, A course of modern analysis, Cambridge University Press, New York, 1973. MR.1424469 (97k:01072)

[30] L.-C. Zhang, q-difference equations and Ramanujan-Selberg continued fractions, Acta Arith. 57 (1991), 307-355. MR 1109991 (92j:11008)

[31] L.-C. Zhang, Ramanujan's class invariants, Kronecker's limit formula and modular equations. II, Analytic Number Theory, Proceedings of a Conference in Honor of H. Halberstam, vol. 2, Birkhäuser, Boston, MA, 1996, pp. 817-838. MR1409396 (97i:11040) 
[32] L.-C. Zhang, Ramanujan's class invariants, Kronecker's limit formula and modular equations. III, Acta Arith. LXXXII.4 (1997), 379-392. MR1483690 (98k:11045)

[33] L.-C. Zhang, Explicit evaluations of a Ramanujan-Selberg continued fraction, Proc. Amer. Math. Soc. 130 (2002), 9-14. MR:1855613 (2002g:11186)

Department of Mathematical Sciences, University of Illinois at Springfield, SpringFIELD, ILLINOIS 62703-5407

E-mail address: chan.hei-chi@uis.edu 